\title{
Ralf Michaels \\ Standortwettbewerb als Rechtsfigur \\ Die Entscheidung des OVG Hamburg zum Airbus A 380 \\ im Lichte der Globalisierungsdebatte
}

\section{Einleitung}

Die im Verfahren des einstweiligen Rechtsschutzes ergangene Entscheidung, mit der das OVG Hamburg grünes Licht für den Ausbau des Flugzeugbauwerks Finkenwerder zugunsten des neuen Airbus A 380 gegeben hat, ${ }^{\mathrm{I}}$ ist von immenser Bedeutung für Stadt und Region Hamburg. Wirtschaftlich erhofft man sich vom Bau durch die EADS eine Vielzahl neuer Arbeitsplätze und eine erhebliche finanzielle Stärkung der städtischen Wirtschaftslage. ${ }^{2}$ Andererseits macht der Ausbau es erforderlich, das Mühlenberger Loch teilweise zuzuschütten, eine Ausbuchtung der Elbe, in der die gefährdete Löffelente brütet und die als Europäisches Vogelschutzgebiet ${ }^{3}$ und po-

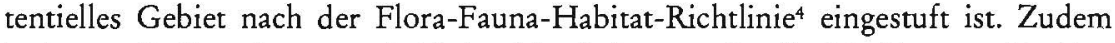
bedeutet die Erweiterung erhebliche Lärmbelastung für die Nachbarn auf beiden Seiten der Elbe. Diese erhebliche Bedeutung der Entscheidung für die Wirtschaft und den Naturschutz sowie die Anwohner war die Ursache für die Heftigkeit, mit der Befürworter wie Gegner des Ausbaues ihren jeweiligen Standpunkt zu Gehör brachten. Das ging teilweise an die Grenzen der gebotenen Zurückhaltung vor der Unabhängigkeit der Justiz; Appelle an die Verantwortung der entscheidenden Richter erreichten in einigen Fällen fast die Stufe des Versuchs der Richterbeeinflussung. Hier soll es nicht darum gehen, inwieweit sich die entscheidenden Richter bei der Abweisung der von Nachbarn eingebrachten Klage einem solchen Druck von einer der beiden Seiten gebeugt haben, denn eine solche Behauptung wäre rein spekulativ. Auch geht es nicht um eine spezifisch verwaltungsrechtliche Kritik am Urteil. Worum es geht ist, exemplarisch aufzuzeigen, ob und wie die außerrechtlichen Argumente von Befürwortern und Gegnern in rechtlichen Argumenten widergespiegelt werden, ob die herkömmliche juristische Begrifflichkeit den sich stellenden Fragen gerecht werden kann, und wie sich all das - und auch das Ergebnis des OVG - in die allgemeine Globalisierungsdebatte einbetten.

I OVG Hamburg, Beschl. v. 19. 2. 200I (2 Bs 370/00), NVwZ 2001, I I73; anders noch die Vorinstanz: VG Hamburg, Beschl. v. I 8.12. 2000 (Is VG 3923/00), NordÖR 200I, 34 m. zust. Anm. Prall; Beschl. v.

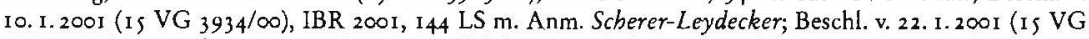
$4838 \% \circ$ ). Die Urteile von VG und OVG sind leicht zugänglich über www.hamburg.de/StadtPol/Gerichte/ gerichte.htm.

2 Die von Gegnern des Projekts aufgeworfenen $Z$ weifel, ob diese positiven wirtschaftlichen Effekte wirklich wahrscheinlich sind, sollen hier unbehandelt bleiben. Dazu Babnsen, Ein Fall für den Rechnungshof, Die Welt vom 21.5.2001.

3 I9a Abs. 2 Nr. 4 BNatSchG; Richtlinie $79 / 409 /$ W WG des Rates vom 2. April 1979 (Vogelschutz-Richtlinie), Abl. EG 1979, L $103 / \mathrm{I}$.

4 Richtlinie 92/34/EWG des Rates vom 21. Mai 1992, Abl. EG 1992, L 206/7; vgl. dazu nur Louis, Die Umsetzung der Fauna-Flora-Habitat-Richtlinie durch das Bundesnaturschutzgesetz und ihre Rechtsfolgen, DÖV 1999, 374-381. Zum Verhältnis zur Vogelflug-Richtlinie Jarass, EG-rechtliche Folgen ausgewiesener und potentieller Vogelschutzgebiete, ZUR 2000, $183-190 ;$ EuGH, Urt. v. 7. I 2. 2000, Rs. C-374/ 98 Kommission ./. Frankreich, DVBI. 2001 , i 56. 


\section{Argumentationsstrukturen}

Aus der Fülle der im Beschluß behandelten Fragen seien drei herausgegriffen: die nach der Gemeinnützigkeit des Planvorhabens, die der Folgenabwägung sowie die nach der Relevanz naturschutzrechtlicher Belange.

\section{a) Gemeinnützigkeit v. Privatnützigkeit}

Seit der Naßauskiesungsentscheidung des BVerwG aus dem Jahre $1978^{5}$ trennt man bei der Frage, inwieweit Planvorhaben in Rechte Dritter eingreifen dürfen, zwischen privatnützigen und gemeinnützigen Vorhaben und sieht eine Rechtfertigungsmöglichkeit nur bei Letzteren. Das OVG läßt die Frage der Gemeinnützigkeit des Ausbaus ausdrücklich offen, gibt aber zu erkennen, daß es einer solchen Einstufung durchaus wohlwollend gegenübersteht, ${ }^{6}$ und will auch für den Fall der Privatnützigkeit das Vorhaben weitgehend wie ein gemeinnütziges behandeln. ${ }^{7}$ Das erscheint zunächst überraschend - schließlich dient das Vorhaben unmittelbar den Gewinninteressen eines privaten Investors, der EADS. Noch 1994 und 1998 hatte ein anderer Senat des OVG den vorigen Ausbau des Werks ausdrücklich als privatnützig bezeichnet. ${ }^{8}$ Nun will das Gericht auch die mittelbaren Folgen des Vorhabens einbeziehen: Verbesserung der regionalen Wirtschaftsstruktur, Schaffung einer nicht unerheblichen Zahl von Arbeitsplätzen, Förderung der Entwicklung von Produkten mit Bedeutung für das Gemeinwesen.

Wirtschaftlich betrachtet ist das richtig, die Implikationen der Argumentation für das Recht sind dagegen problematisch. Unausgesprochen liegt ihr die schon von Adam Smith formulierte Einsicht zu Grunde, daß das Aufeinandertreffen der Individualinteressen im Wettbewerb letztlich zum Wohle aller führen wird. Andererseits liegt danach der einzige Zweck des Staates nach liberalem Verständnis im Schutz der Individualinteressen. So gesehen, ist aber im Liberalismus jedes Individualinteresse mittelbar auch ein öffentliches Interesse, jedes öffentliche Interesse mittelbar auch ein privates. Damit verliert das Gegensatzpaar Gemeinnützigkeit - Privatnützigkeit seine Aussagekraft, weil kein Unterschied mehr besteht - jeder der beiden Begriffe geht im jeweils anderen auf. Das erkennt auch das OVG, wenn es die Gefahr nennt, daß »jede Großinvestition eines Wirtschaftsunternehmens, die in der Regel derartige Folgen hat, allein damit den Charakter eines gemeinnützigen Vorhabens gewinnen « könnte, und als Folge "zusätzliche qualifizierende Merkmale entscheiden lassen will. ${ }^{9}$ Es ist aber fraglich, ob sich diese wirklich objektiv gewinnen lassen: Wenn vom argumentativen Ausgangspunkt her zwischen Privat- und Gemeinnützigkeit schon begrifflich kein Unterschied besteht, dann kann ein solcher auch nicht durch die Erfüllung solcher Merkmale ans Licht gebracht werden. Vielmehr beruht dann die Gemeinnützigkeit notwendig auf der gerichtsfreien Bestimmung durch die Verwaltung - was das OVG selbst andeutet. Das Bundesverfassungsgericht hatte dieses Problem in seiner Boxberg-Entscheidung bereits gesehen und jedenfalls im Rahmen von Enteignungen für solche mittelbar gemeinnützigen Vorhaben verlangt, daß das Gemeinwohl gesetzlich konkret definiert und durch das Planvorhaben tatsächlich und

\footnotetext{
5 BVerwG, Urt. v. 10. 2.1978 (4 C 25.75), BVerwGE 55, 220, $226 \mathrm{ff}$

6 OVG Hamburg (Fn. I), unter Il.A.I.

7 OVG Hamburg (Fn. I), unter II.A.2.

8 OVG Hamburg, Beschl. v. I 3. I 2. 1994 (Bs III 376/94), DVBl. I995, 1026 (Ls.); OVG Hamburg, Urt. v. 2.3. 1998 (Bf III 41/96) (aufrechterhalten durch BVerwG 7. I 2. 1998 [ [ I B 46/98], UPR 1999, 153 = ZLW 1999, 533 = NUR 2000, 36); ebenso noch VG Hamburg 18. I 2. 2000 (Fn. I) unter Il.3. 2. 9 OVG Hamburg (Fn. I), unter II.A.I.c.
} 
dauerhaft erreicht wird. ${ }^{10} \mathrm{Ob}$ und inwieweit diese Kriterien auch unterhalb der Enteignungsschwelle zu beachten sind, meinte das OVG im Eilverfahren nicht entscheiden zu können und zu müssen. ${ }^{\text {II }}$

Noch ein weiterer Punkt ist bemerkenswert: daß nämlich offenbar nur Großinvestitionen mittelbar gemeinnützig sein sollen, nicht aber kleine Investitionen. Das wirft die Frage auf nach dem qualitativen Unterschied zwischen der EADS, die 2000 neue Arbeitsplätze schafft, und dem Mittelständler, der nur zwei schafft - bzw. 1000 Mittelständlern, die mit Einzelprojekten jeweils zwei neue Arbeitsplätze schaffen.

\section{b) Unternebmens- und Standortinteressen v. Anwohner- und Verbandsinteressen}

Ein zweites Element betrifft die Abwägung der Nachteile der sofortigen Vollziehung für die Kläger gegen die Nachteile eines Baustopps bis zur Entscheidung des Hauptsacheverfahrens. Das OVG meint, die Interessen der Antragsteller müßten zurückstehen: Während bei ihnen persönlich (also nicht für die Natur) bis zum Hauptsacheverfahren voraussichtlich keine irreparable Rechtsbeeinträchtigung eingetreten wäre, ließe sich andererseits »das Vorhaben « voraussichtlich endgültig nicht realisieren, wenn die aufschiebende Wirkung der Anfechtungsklage aufrechterhalten würde. ${ }^{12}$ Der Grund: Die EADS hatte bereits glaubhaft angekündigt, bei einer Verlängerung des Baustopps die Investition in Hamburg aufzugeben und die Produktion (auch insoweit) nach Toulouse zu verlegen.

Die Argumentation des OVG ist indes nicht unproblematisch: Für die EADS als Beigeladene bedeutet der Aufschub nämlich nach ihrer eigenen Argumentation offenbar gerade keine schweren Nachteile, denn sie hat eine einfache Handlungsoption, die Produktion in Frankreich. Offensichtlich kommt es entscheidend auf die Nachteile für die Stadt Hamburg als Antragsgegnerin an, für die sich diese Handlungsoption nicht stellt - die Arbeitsplätze in Toulouse helfen ihr nicht. Die Interessen der Hansestadt können nun aber strenggenommen nur dann eine Rolle spielen, wenn es sich um ein gemeinnütziges Vorhaben handelt; genau diese Frage hatte das OVG aber zuvor als entscheidungsunerheblich offengelassen.

Dieses Problem läßt sich auch anders darstellen: $\mathrm{Daß}$ sich »das Vorhaben" endgültig nicht realisieren läßt, stimmt nur dann, wenn man den Begriff eng definiert, nämlich konkret den Ausbau des Werks in Hamburg. Im weiteren Sinne ist das Vorhaben durchaus zu realisieren - nur eben in Toulouse. Daß das Gericht diese Möglichkeit nicht mit einbezieht, findet seine Parallele in der herrschenden Ansicht, die sowohl allgemein im Planungsrecht ${ }^{13}$ als auch konkret im Umweltschutzrecht ${ }^{14}$ die Alternativenprüfung geographisch auf die nähere Umgebung begrenzt. Zugespitzt bedeutet das: Der Investor kann zwar weltweit nach Alternativen suchen; von der Verwaltung oder von klagebefugten Anwohnern braucht er sich aber nur auf geographisch naheliegende Alternativen verweisen zu lassen. $\mathrm{Da}$ d das zu einem Ungleichgewicht führen kann, liegt auf der Hand.

10 BVerfG, Urt. v. I6. I 2. 1986 (I BvR 1046/8s), BVerfGE 74, 264, $28_{4}$ ff.

I I Kritisch dazu Prall, Zur Abgrenzung von gemein- und privatnützigen Planfeststellungen, NordÖR 200 , $187-190$.

12 OVG Hamburg (Fn. I), unter II.C.I.

13 Dazu Forschbach, Die Pflicht zur Standortalternativenprüfung in der Planfeststellung und in vorgelagerten Verfahren, 1998.

14 Erbguth, Naturschutz und Europarecht: Wie weit reicht die Pflicht zur Alternativenprüfung gem. Art. 6 Abs. 4 der Habitatrichtlinie?, DVBI 1999, 588-595. 
Den Kern der politischen Debatte um das Bauvorhaben bildete nicht der Nachbarschutz, sondern der Naturschutz. Diesen ließ das OVG unberücksichtigt, und zwartrotz der scharfen Kritik von Naturschutzverbänden - wohl entsprechend dem geltenden Recht. Belange des Naturschutzes berühren keine subjektiven Rechte der Kläger, folglich blieben sie gleich an zwei Stellen außer Betracht: Für die Frage der Rechtmäßigkeit wurden Verstöße gegen Naturschutzrecht nicht geprüft. ${ }^{\text {'s }}$ Aber auch bei der Folgenabwägung blieben die eventuell irreparablen Naturschäden außer Betracht, denn "auch die an den Vollzugsfolgen orientierte Interessenabwägung im Verfahren nach $\$ 80$ Abs. s VwGO ist auf die Berücksichtigung der den Antragstellern zustehenden subjektiv-öffentlichen Rechte beschränkt «. ${ }^{16}$ Die parallel von $\mathrm{Na}$ turschutzverbänden angestrengten Verbandsklagen waren schon vom VG zurückgewiesen worden: Beteiligungsrechte seien nicht verletzt worden, und die eng umrissenen Voraussetzungen von $\$ 4$ I $\mathrm{HmbNatSchG} \mathrm{für} \mathrm{die} \mathrm{Verbandsklage} \mathrm{seien} \mathrm{nicht}$ erfüllt; auch aus Gemeinschaftsrecht lasse sich keine Klagebefugnis folgern. ${ }^{17}$

Das ist, nach herkömmlicher Verwaltungsrechtslehre, wohl richtig. Das eigentliche Problem ist damit aber noch nicht einmal angesprochen. Daß der Naturschutz hier mangels Prüfbarkeit zurückstehen muß, beruht nämlich auf einem erstaunlichen Positionstausch: Während die privaten Interessen der EADS, jedenfalls tendenziell, im Rahmen des Gemeinwohls in öffentliche übersetzt werden (und dadurch den Individualinteressen der Nachbarn vorgehen) können, gelingt es andererseits den Antragstellern nicht, öffentliche Interessen - namentlich den Naturschutz - in private zu übersetzen, die im Rahmen des $\$ 42$ Abs. 2 VwGO relevant wären. So ist es etwas pikant, wenn die Kläger einerseits von der Politik aufgefordert werden, ihren Egoismus dem Gemeinwohl unterzuordnen, während sie andererseits, soweit sie versuchen, Gemeinwohlinteressen vorzubringen, vor Gericht nicht gehört werden.

\section{Die Globalisierungsdebatte als Instrument zur Erklärung des OVG-Beschlusses}

\section{a) Themen der Globalisierung}

Was hat das alles nun mit Globalisierung zu tun? Die Globalisierungsdebatte stellt hergebrachte Grundlagen des Rechts in Frage: Einerseits wird die Bedeutung des Staates als souveräner Regelungsinstanz relativiert, andererseits nähern sich große multinationale Unternehmen allein schon wegen ihrer wirtschaftlichen Macht politisch im Bedeutungsgrad Staaten an. ${ }^{18}$ Damit verbunden ist auch eine Durchmischung von öffentlichem und privatem Bereich - nicht nur beeinflussen beide einander, vielmehr werden sie, jedenfalls in ihrer herkömmlichen Definition, ununterscheidbar.

Is OVG Hamburg (Fn. I) unter II.B.2.b.

I6 OVG Hamburg (Fn. I) unter II.C. 3 .

17 VG Hamburg, Beschl. v. Is. I. 2001 (15 VG 3932/00); bestätigt durch OVG Hamburg, Beschl. v. 27. 2. 2001 (Bs 38/0I), und durch BVerfG, Beschl. v. IO. 5.200I (I BvR 48I/0I, 5I8/0I), NordÖR 2001, 261 = DVBI. 200 I, I I 39. Zu Klagerechten von Umweltverbänden zuletzt Ziekow/Siegel, Anerkannte Naturschutzverbände als $*$ Anwälte der Natur $*, 2000,95-1$ 20; Hoppe/Beckmann/Kauch, Umweltrecht ${ }^{2}$, 2000, \$I Rn. 84-90; weitere Nachweise bei Kopp/Schenke, VwGO'z, 2000, $\$ 42$ Rn. I 8I Fn. 419. Mittlerweile hat Hamburg in einer Novelle zum Naturschutzgesetz die Klagerechte von Naturschutzverbänden erweitert, Großprojekte wie der Flugzeugbau sind aber weiterhin davon ausgenommen.

18 Vgl. nur Friedmann, The Lexus and the Olive Tree, 2. Aufl. 2000, deutsch: Globalisierung verstehen Zwischen Marktplatz und Weltmacht, 2000; früher schon etwa Dezalay Marchands de droit - La restructuration de l'ordre juridique international par les multinationales du droith 1992; David J. Saari, Global corporations and sovereign nations: collision or cooperation?, 1999 . 
Die Machtangleichung zwischen multinationalen Unternehmen und Staaten läßt sich auch anders beschreiben: Durch die Vernetzung der Welt entstehen für mobile Faktoren - insbesondere Kapital, daneben aber auch etwa Kapitalgesellschaften vorher ungekannte Entscheidungsspielräume; andererseits sehen sich lokale Faktoren - Wirtschaftsstandorte, Naturschutzgebiete - auf einmal im Wettbewerb mit gleichartigen Faktoren in anderen Ländern, der ihnen neue Zwänge auferlegt; der Staat als notwendig lokale Einheit wird in seiner Regelungsfreiheit faktisch beschränkt. ${ }^{19}$ Was das für den Naturschutz bedeutet, ist umstritten: Einige meinen, Volkswirtschaften seien frei in der Setzung ihrer Umweltstandards, nur müßten sie diese gegen Lohneinbußen abwägen ${ }^{20}$ andere glauben, daß im freien Markt kurzfristige Unternehmensgewinne die Überhand gegenüber erst in der Zukunft spürbaren Umweltschädigungen haben werden (wobei das die Frage aufwirft, wie sich außerhalb des Marktes der »eigentlich« höhere Wert der Umwelt objektiv messen läßt). Sicher ist wohl jedenfalls, daß wirtschaftliche Interessen unmittelbar (durch ihre Träger), solche des Umweltschutzes dagegen nur mittelbar (durch Wahlen oder Konsumverhalten) zur Geltung kommen.

Schließlich ist zwar Globalisierung nicht mit Ökonomisierung gleichzusetzen; wohl aber zeigt sich, daß ökonomische Überlegungen und Bewertungen wesentlich mehr Gewicht erlangen. Das ist auch eine Folge dessen, daß die Macht multinationaler Unternehmen primär auf ihrer Investitionsfähigkeit beruht.

$\mathrm{Daß}$ sich alle diese Elemente im Streit um das Mühlenberger Loch wiederfinden lassen, ist offensichtlich. Juristisch interessant ist erst, wie sie sich auch in der rechtlichen Begründung des OVG widerspiegeln, wie sie es verständlicher machen.

\section{b) Privatisierung des Öffentlichen}

Aus Sicht der Globalisierungsdebatte wird verständlich, wie sowohl die Tendenz des OVG, das Bauvorhaben als gemeinnützig einzuordnen, als auch die angesprochene Verwandlung privater Interessen in öffentliche im Rahmen der Interessenabwägung die oben angesprochene politische Vermischung des öffentlichen und privaten Bereichs in die rechtliche Beurteilung übertragen. Tatsächlich stehen sich nämlich die Stadt Hamburg und die EADS nur aus der Nahperspektive in dem für das öffentliche Recht charakteristischen Über-Unterordnungsverhältnis gegenüber. Aus globaler Perspektive dagegen verschiebt sich das Gewicht zu Gunsten der EADS und zu Lasten der Stadt. Privatinteressen der EADS und öffentliches Interesse der Stadt Hamburg stehen auf einer Stufe; das Recht vollzieht diese Gleichstellung nur nach.

Die Boxberg-Entscheidung des Bundesverfassungsgerichts erscheint so in einem neuen Licht: Im darin aufgestellten konkreten Gesetzesvorbehalt liegt die zutreffende Folgerung daraus, daß es sich bei der Abgrenzung von Gemein- und Privatnützigkeit um eine nicht begrifflich zu klärende Frage handelt: Folglich ist sie politisch zu entscheiden, also vom Parlament. (Inwieweit das Parlament diese Fragen noch frei entscheiden kann, ist freilich eine andere Frage.) Problematischer sind die weiteren aufgestellten Kriterien - Sicherheit und Dauerhaftigkeit der Gemeinwohlförderung. Sie beruhen auf dem Bild des Staates als übergeordnetem Wirtschaftslenker. Sieht man ihn dagegen selbst als Teilnehmer am (Standort-) Wettbewerb, so handelt er notwendig unter der gleichen Unsicherheit, ob sich seine Investitionen rentieren werden, wie ein Privatunternehmen. Die scharf formulierte Forderung, Entscheidungen in

19 Von einer "global hierarchy of mobility* spricht etwa Bauman, Globalization - The Human Consequences, I998, 69-76.

20 von Weizsäcker, Logik der Globalisierung, 1999, 71-76. 
diesem Gebiet dürften nicht justitiabel sein, ${ }^{21}$ zieht die Konsequenz aus dieser Einsicht, ohne allerdings deren Folgen für das Konzept des Rechtsstaats wirklich auszuloten.

Im übrigen wird nun auch die oben angesprochene Privilegierung von Großinvestoren gegenüber Kleininvestoren aus Sicht der Globalisierung verständlich: Nur der Großinvestor hat nämlich weltweite Alternativen. ${ }^{22} \mathrm{Zwar}$ machen sich auch Kleininvestoren einen Standortwettbewerb zunutze, etwa zwischen der Stadt Hamburg und Orten des sogenannten Speckgürtels« in den angrenzenden Ländern, SchleswigHolstein und Niedersachsen. Auf diesen Standortwettbewerb kann das Recht aber evtl. noch reagieren: In der näheren Umgebung kann es im Rahmen von Planungsalternativen einen Standort den anderen vorziehen, sofern Rechte Dritter oder der Naturschutz betroffen sind. Auf die Prüfung europaweiter oder gar weltweiter Standortalternativen ist das Recht dagegen nicht eingerichtet. Es ist auch sehr fraglich, ob eine solche Prüfung faktisch durchsetzbar wäre. Die Legitimität etwa der EU stünde wohl in Frage, wenn sie eine solche Prüfung durchführte oder verlangte, anstatt aus politischen Gründen eine gleichmäßige Beteiligung der Mitgliedstaaten an einem Großprojekt wie dem Airbus-Bau zu fordern. Weltweiter Standortwettbewerb ist bislang durch das Recht nur schwer zu regulieren.

\section{c) Ökonomisierung des Rechts}

Die Zurückstellung der Rechte der Nachbarn scheint dem der Globalisierungsdebatte zugrunde liegenden Liberalismus mit seiner Betonung individueller Rechte, insbesondere des Eigentums, zu widersprechen. Aber das betrifft vielleicht nur den klassischen Liberalismus. Daß die Interessen der Nachbarn gegenüber denen der EADS zurückstehen müssen, läßt sich nämlich ökonomisch mit Effizienzüberlegungen begründen: Das Recht, Lärm zu erzeugen, ist für die EADS mehr wert als das Recht auf Lärmfreiheit für die Anwohner, weil die EADS mehr dafür bezahlen könnte (und - vielleicht - würde). Dann ist es aber, so die Theorie, effizienter, der EADS das Recht auf Lärm zuzusprechen. Ob die Anwohner Anspruch auf Ausgleichszahlungen haben, wie es das OVG erwägt, ist für die Allokationseffizienz irrelevant und läßt sich nur aus Distributionsgesichtspunkten begründen.

Die Kosten für die Natur sind dagegen externe Kosten und als solche traditionell schwer in eine an Angebot- und Nachfragepreis orientierte Effizienzprüfung einzubeziehen. So bekommt auch das Argument des $\$ 42$ Abs. 2 VwGO, daßindividuelle Kläger nicht Belange des Naturschutzes vorbringen können, eine neue Brisanz. Es fügt sich nämlich nun in die Debatte ein, ob in der Globalisierung Interessen, die sich nicht unmittelbar in individuell wirtschaftliche umsetzen lassen, verloren gehen, weil sie vom Staat als dem Vertreter des Gemeinwohls nicht mehr in gleichem Maße wie zuvor geschützt werden können. Wenn etwa gegen ein Verbandsklagerecht anführt wird, der Schutz überindividueller Interessen müsse in der Demokratie der allgemeinen politischen Willensbildung überlassen werden, ${ }^{23}$ so ist das nur dann überzeugend, wenn die entscheidenden Fragen noch durch Wahlen und Abstimmungen entschieden werden können - genau das wird aber von Theoretikern der Globalisie-

2I Voscherau, Die »Vetokratie« muß reformiert werden, Die Welt vom I g. I. 200I: »Im gewaltengeteilten Verfassungsstaat gehören Weichenstellungen für die Zukunft, die über Wohl und Wehe eines Volkes oder ganzer Regionen entscheiden können, vor Parlament und Regierung, nicht vor die dritte Gewalt. Denn demokratische Legitimation für solche Weichenstellungen und politische Ergebnisverantwortung für Gelingen oder Scheitern liegen in der Demokratie nur bei den ersten beiden Gewalten.« Jedenfalls für die zweite Gewalt, die Verwaltung, erscheint das zweifelhaft.

$22 \mathrm{Vgl}$. Beck, Was ist Globalisierung?, 1997, $2 \mathrm{I}$.

23 Kloepfer, Umweltrecht 2 , 1998, $\$ 8$ Rn. 31 . 
rung mehr und mehr in Frage gestellt. ${ }^{24}$ In den USA wird schon lange erwogen, ob Gemeinwohl und Recht nicht statt durch Wahlen effektiver durch Klagen durchgesetzt werden können; das wird sogar auf die Außenpolitik bezogen. ${ }^{25}$ In Deutschland steht man einer solchen Entwicklung noch kritisch gegenüber und hofft auf den Staat, wobei man nicht immer die Einschränkung seines Handlungsspielraums berücksichtigt. So kann es nicht verwundern, daß die vielleicht wirksamste Opposition gegen die Pläne eines weltweit tätigen Unternehmens von einer ihrerseits weltweit tätigen Nichtregierungsorganisation betrieben wird (IFAW - International Fund for Animal Welfare), die versucht, durch weltweite Anzeigenkampagnen in Tageszeitungen und das Internet Druck zu machen. ${ }^{26}$

Die ökonomische Analyse des Rechts als Unterordnung des Rechts unter ökonomische Strukturen und das normative Ziel der Allokationseffizienz ist zwar als Theorie schon lange bekannt. ${ }^{27}$ In der deutschen Rechtsprechung, zumal im Verwaltungsrecht, hat sie dagegen bislang wenig Beachtung gefunden. Die Entscheidung des OVG Hamburg mag nun ein Anzeichen dafür sein, daß Gerichte, wenn auch unbewußt, unter dem Druck der Globalisierung faktisch doch mehr und mehr gemäß dem Postulat ökonomischer Theorie urteilen werden. Das wäre ein Beleg für die in den USA vertretene These, daß Richterrecht wie von selbst mehr und mehr zu ökonomisch effizienten Lösungen strebt. Die Frage nach der normativen Berechtigung einer solchen Entwicklung ist damit indes nicht beantwortet, nicht einmal reflektiert. Einer "zufällig « an Effizienzkriterien ausgerichteten Rechtsprechung dürfte aber jedenfalls eine solche vorzuziehen sein, die sich ihrer Kriterien bewußt ist.

\section{Ausblick: Globalisierung und Legitimation}

Es sei noch einmal betont: Weder eine spezifisch verwaltungsrechtsdogmatische, noch eine politische oder gar moralische Kritik war mit diesem Beitrag beabsichtigt. Für ersteres wären weitaus differenziertere Auseinandersetzungen mit dem Fachrecht erforderlich. Letzteres würde möglicherweise die Zwänge verkennen, welche die Globalisierung mit sich bringt: Die Verantwortlichen der Stadt Hamburg sind schließlich für das Investitionsklima ihrer Stadt verantwortlich. Es liegt dagegen nahe, der EADS vorzuwerfen, daß sie Hamburg gegen Toulouse (und Rostock) ausgespielt hat, um ihre Position zu verbessern. Indes griffe auch dieser Vorwurf zu kurz: Auch die EADS steht natürlich im weltweiten Wettbewerb ${ }^{28}$ und ist darauf angewiesen, möglichst kostengünstig zu produzieren. In gewissem Ausmaß gilt das schließlich auch für die EU, die den Bau, nach anfänglichem Zögern, als naturschutzrechtsgemäß genehmigt hat; ${ }^{29}$ sie weiß von der Bedeutung des Airbus für die europäische Wirtschaft. Daß in diesem globalen Wettbewerb Nachbarn und Löffelente das Nachsehen haben, ist fast zwangsläufig, man kann es daher-so scheint es beinahe-den Akteuren nicht vorwerfen.

Von einer höheren Warte aus betrachtet, liegt allerdings vielleicht gerade hier das Problem. Die Globalisierung in ihrem herkömmlichen Verständnis führt zum Vorrang des Ökonomischen vor dem Politischen. Damit verbunden ist der Verlust von

24 Vgl. nur McGrew (Hrsg.), The Transformation of Democracy? Globalization and Territorial Democracy, 1997.

2s Slaughter/Bosco, Plaintiff's Diplomacy, Foreign Affairs September/October 2000, 102-1 16.

26 www.airbusthinktwice.com. Die EADS selbst informiert aus ihrer Sicht unter www.airbus-a380.de.

27 Vgl. nur Eidenmüller, Effizienz als Rechtsprinzip², 1998; Kirchner, Ökonomische Theorie des Rechts, 1997 .

28 Vgl. nur „EADS: >Monopol der Amerikaner brechen««, WirtschaftsWoche heute vom 16.5.200I, www.wiwo.de.

29 Vorbehaltlich der Schaffung von Ausgleichsflächen. 
Verantwortung, sowohl des Staates als auch der Privaten. Wenn alle nur noch Getriebene des Wettbewerbs sind, handeln letztlich alle unter Zwang, können Entscheidungen nur in einem enger werdenden Rahmen getroffen werden. Die Entscheidung des OVG zeigt, wie das sich letztlich auch auf die Gerichte als Rechtsanwender auswirkt, wie sich diese allgemeinen Erkenntnisse in konkreten Gerichtsentscheidungen wiederfinden lassen und diese beeinflußen.

Die Frage, wie das Recht auf diese Entwicklungen reagieren soll, ist noch offen. Man kann das Recht der Ökonomie unterordnen oder mit seiner Hilfe einen heroischen (und vielleicht zwecklosen) Kampf gegen sie zu führen versuchen; man kann die Lösung der Regulierungsprobleme in einem Weltrecht suchen oder auf Regulierung ganz verzichten; man kann nach ganz neuen Formen des Rechts suchen. ${ }^{30}$ Wie konkrete Rechtsfragen dann gelöst werden, ob man der Löffelente und den Nachbarn die Wirtschaftsinteressen vorziehen sollte oder nicht, das ist eine Frage, auf die man in jedem Bezugsrahmen verschiedene Antworten finden kann. Sicher ist wohl nur eins: Wenn das Recht unfähig werden sollte, schon die zur Beantwortung dieser Frage adäquaten Argumente zur Verfügung zu stellen, weil seine Methodik den Entwicklungen nicht mehr entspricht, dann ist seine Legitimität zweifelhaft geworden. Hierin liegt - unabhängig vom Ergebnis - das wirklich Beunruhigende der Entscheidung des OVG.

\begin{tabular}{|l|}
\hline Birgit Harbeck \\
\hline Probleme des Einheitstatbestandes \\
sexueller Nötigung/Vergewaltigung \\
\hline \\
\hline $\begin{array}{l}\text { Durch die Reform des } \$ \S 177 \text { ff. StGB wurden die Straftatbestände der } \\
\text { Vergewaltigung und der sexuellen Nötigung zu einem vorläuffigen } \\
\text { Abschluß gebracht. Das Werk zeigt Regelungen und Probleme auf, die } \\
\text { durch den neu gefaßten Einheitstatbestand entstanden sind. }\end{array}$ \\
$\begin{array}{l}\text { 2001, } 238 \text { S., brosch., } 40,-€, 68,50 \text { sFr, } 1 S B N \text { 3-7890-7107-2 } \\
\text { (Kieler Rechtswissenschaftliche Abhandlungen (NF), Bd. 29) }\end{array}$ \\
\hline NOMOS Verlagsgesellschaft \\
$\mathbf{7 6 5 2 0}$ Baden-Baden
\end{tabular}

30 Aus den zahlreichen Forschungsansätzen in diesem Bereich sei nur genannt Güntber/Randeria, Recht, Kultur und Gesellschaft im Prozeß der Globalisierung - Werner Reimers Stiftung, Schriftenreihe Suchprozesse für innovative Fragestellungen in der Wissenschaft Heft 4 (200I), im Internet unter www.suchprozesse.de. 